\title{
UEMS: Fokus auf ärztlicher Weiter- und Fortbildung sowie Qualitätssicherung
}

\section{Max Giger}

Past-Präsident Schweizerisches Institut für ärztliche Weiterund Fortbildung SIWF
Zum 52. Mal lud die Union Européenne des Médecins Spécialistes (UEMS) zu ihrer Hauptversammlung ein. Diese fand vom 7. bis 9. Oktober 2010 in Prag statt und war von der Tschechischen Ärztegesellschaft organisiert. Erstmalig tagten die Vertreter der nationalen Ärztegesellschaften («Council») gemeinsam mit denjenigen der wissenschaftlichen Fachgesellschaften («Sections and Boards»). Als wichtigste Traktanden verabschiedeten sie die in den letzten fünf Jahren erarbeitete Statutenrevision - sie trägt dem engeren Zusammengehen der nationalen Ärzteorganisationen und der Fachgesellschaften Rechnung - und den Kauf einer Domus medica im EU-Quartier in Brüssel.

Mit diesen Beschlüssen stärkt die UEMS ihre europäisch anerkannte Stellung als Wegbereiterin der ärztlichen Weiter- und Fortbildung sowie der Versorgungsqualität von Patienten. Ein «Standing Committee» zur Weiterbildung wurde geschaffen, ebenso wie eines zur Fortbildung und ein weiteres zum Qualitätsmanagement. den - dies im Gegensatz zur aktuellen politischen Tendenz der Nivellierung nach unten. Erste Resultate der drei Pilot-Fachgesellschaften (Radiologie, Kardiologie, Anästhesiologie) sind auf Mitte 2011 zu erwarten. Die Verleihung der Facharztdiplome folgt nationalem Recht. Die Mitgliedsländer sind zur Kooperation eingeladen. Die skandinavischen Länder begrüssen ein formatives Assessment der ärztlichen Kompetenzen, sie lehnen jedoch ein summatives ab. Die Arbeit an den schon bestehenden 27 europäischen Facharztassessments («European Boards») soll wie bisher durch das CESMA fortgesetzt werden. Das erfolgreiche Absolvieren von European Boards ist u.a. integraler Bestandteil der Schweizer Facharztprüfungen für Ophthalmologie, Anästhesiologie, Plastische, Rekonstruktive und Ästhetische Chirurgie und Urologie.

Das Fortbildungskomitee vereinigt die bestfunktionierende Akkreditierungsinstanz für ärztliche Fortbildung (European Accreditation Council for Conti-

\section{Mit diesen Beschlüssen stärkt die UEMS ihre europäisch anerkannte Stellung als Wegbereiterin der ärztlichen Weiter- und Fortbildung}

Das CESMA (Council for European Speciality Medical Assessments), die bisherige Arbeitsgruppe Weiterbildung und die künftige internetbasierte Weiterbildungsplattform für Akkreditierung von Wissen, Fertigkeiten und Professionalität (European Council for the Accreditation of Medical Specialist Qualification/ECAMSQ) konstitutieren das Weiterbildungskomitee. Dank dieser Zusammensetzung lassen sich die Anstrengungen für die europäische Akkreditierung der ärztlichen Weiterbildung bzw. der europäischen Facharztprüfungen bündeln. Das Assessment des Wissens erfolgt über Multiple-Choice-Prüfungen. Fertigkeiten werden formativ - wie in Grossbritannien, den Niederlanden und der Schweiz - mittels arbeitsplatzbasierten Assessments [1] erfasst. Das Assessment der Professionalität der Kandidatinnen und Kandidaten erfolgt ebenfalls dezentral. Durch das ECAMSQ soll ein hoher europäisch anerkannter Standard für die Kompetenz der Fachärzte erreicht wer- nuing Medical Education/EACCME) und die bisherige Arbeitsgruppe. Dieses Komitee führt die auf der Deklaration zur ärztlichen Fortbildung der europäischen Fachärztinnen und Fachärzte von 1994 (Charter on CME) sowie der Basler Deklaration (2001) basierenden Anstrengungen für eine kontinuierliche berufliche Weiterentwicklung (Continuous Professional Development/CPD) fort.

Eine hohe und patientengerechte Qualität der medizinischen Versorgung ist das erklärte Ziel des Komitees für Qualitätsmanagement. Einsitz in diesem Komitee haben das European Advisory Council for Quality Management of Specialist Medical Practice (EACQM) und die bisherige Arbeitsgruppe.

Die Mandatsdauer in allen UEMS-Gremien ist nun einheitlich geregelt: Sie umfasst vier Jahre und kann einmalig verlängert werden. Diese Neuregelung ist ab der nächsten Wahlperiode gültig. Nichts geändert hat sich bei der Wahl der Ländervertreter: Sie 


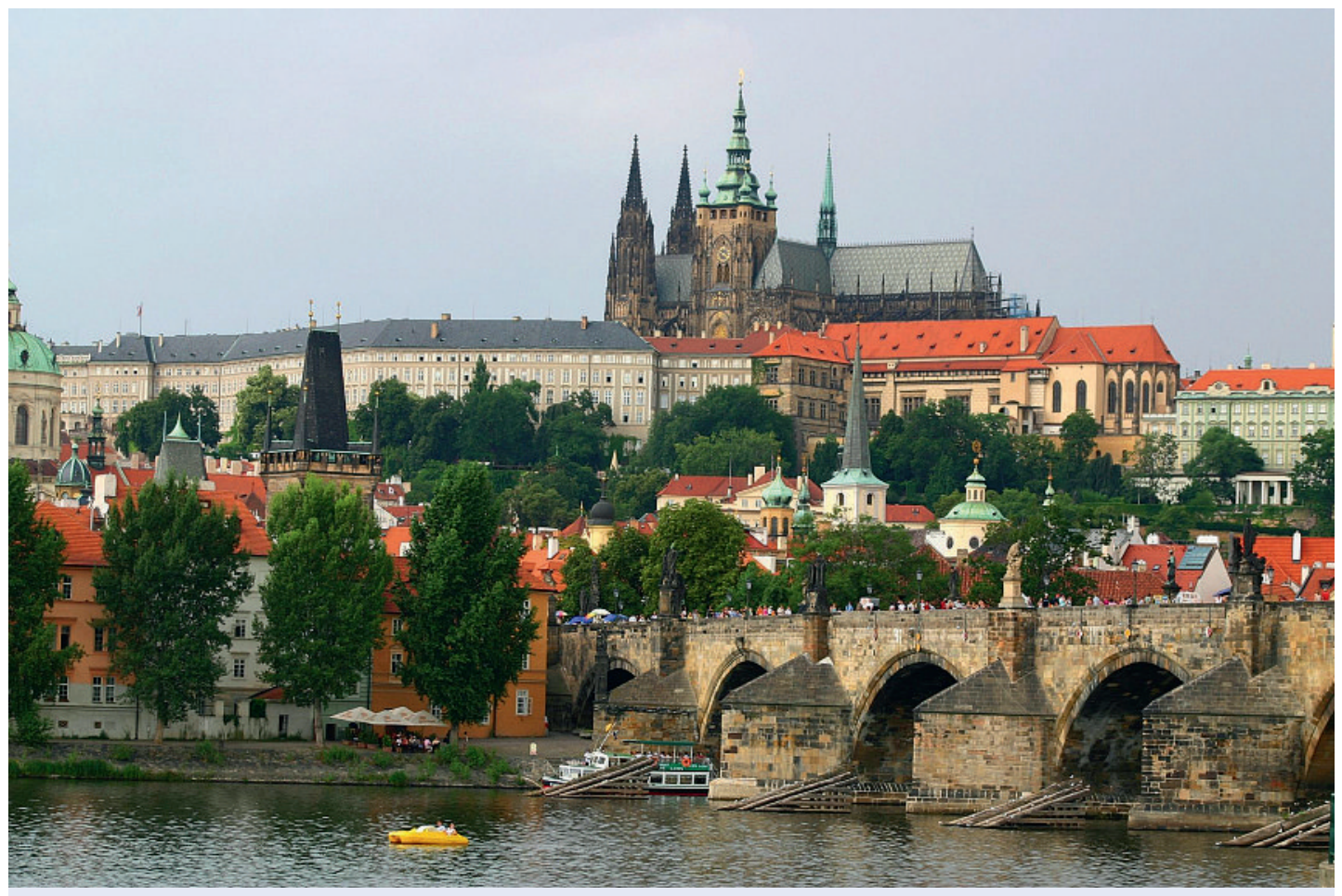

Die Hauptversammlung der Union Européenne des Médecins Spécialistes (UEMS) fand dieses Jahr in Prag statt.

werden wie bisher durch die Mitgliederländer bestimmt und damit für die Dauer von deren Präsenz.

Um den Fachgesellschaften die enge Kooperation innerhalb der UEMS zu erleichtern und die seit über einem Jahrzehnt angestrebte engere Kooperation mit den anderen Europäischen Ärzteorganisationen

\section{Nur wenn sich die europäischen Ärzteorganisationen enger zusammenschliessen und zu einer Stimme der Ärzteschaft werden, können sie in Brüssel erfolgreich verhandeln}

können sie in Brüssel erfolgreich verhandeln. Die UEMS ist gerne bereit, die bildungs- und qualitätsrelevanten Interessen der anderen Gesellschaften, namentlich der UEMO und der PGW, aufzunehmen. Der Vertreter der UEMO wies auf interne Diskussionen hin, die eine Integration der UEMO als Fachgesellschaft für Hausarztmedizin in die UEMS als Fernziel haben.

In der Handchirurgie und der Notfallmedizin beschloss die UEMS die Schaffung von europäischen Diplomen («European Boards»).

Der Fokus der 52. UEMS-Hauptversammlung richtet sich einerseits auf eine qualitativ hochstehende ärztliche Versorgung von Patientinnen und Patienten. Andererseits setzt sich die UEMS als älteste europäische Ärzteorganisation noch stärker für eine möglichst hohe Qualität der ärztlichen Weiter- und Fortbildung in allen Ländern Europas ein. Nur so kann die gegenseitige Anerkennung der Facharztdiplome innerhalb Europas im Interesse der Patientinnen und Patienten ohne Abstriche an Sicherheit und Qualität erfolgen.

1 Montagne S, Jucker-Kupper P, Berendonk C, Rogausch A, Beyeler C, Giger M. Drei Jahre Erfahrung mit Arbeitsplatzbasiertem Assessment (Mini-CEX und DOPS) in der ärztlichen Weiterbildung. Schweiz Ärztezeitung. 2010;91(4):109-11. schen Ärzteorganisationen enger zusammenschliessen und zu einer Stimme der Ärzteschaft werden,

(Union Européenne des Médecins Omnipracticiens EMO, Europäische Arbeitsgemeinschaft der Niede enen Ärzte/EANA, Fédération Européen

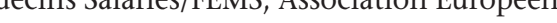
Group of European Junior Doctors/PGW, Comité Permanent des Médecins Européens/CPME) zu ermöglichen, will man eine Liegenschaft im EU-Quartier von Brüssel erwerben. Denn nur wenn sich die europäi- 